\title{
Rivaroxaban for Stroke Prevention in Patients with Non-Valvular Atrial Fibrillation and Active Cancer
}

\author{
Eva S Laube, MDa, Anthony Yu, MD ${ }^{\mathrm{b}}$, Dipti Gupta, MD, MPH ${ }^{\mathrm{b}}$, Yimei Miao, BS ${ }^{\mathrm{a}}$, Patrick \\ Samedy, MS $^{c}$, Jonathan Wills, BA ${ }^{d}$, Stephen Harnicar, PharmD ${ }^{e}$, Gerald A. Soff, MD $^{a}$, and \\ Simon Mantha, MD, MPH ${ }^{\mathrm{a}}$ \\ aHematology Service, Memorial Sloan Kettering Cancer Center, 1275 York Avenue, New York, NY \\ 10065 USA \\ ${ }^{\mathrm{b} C a r d i o l o g y}$ Service, Memorial Sloan Kettering Cancer Center, 1275 York Avenue, New York, NY \\ 10065 USA \\ ${ }^{\circ}$ Quality and Safety, Memorial Sloan Kettering Cancer Center, 1275 York Avenue, New York, NY \\ 10065 USA \\ dInformation Systems, Memorial Sloan Kettering Cancer Center, 1275 York Avenue, New York, \\ NY 10065 USA \\ ePharmacy Department, Memorial Sloan Kettering Cancer Center, 1275 York Avenue, New York, \\ NY 10065 USA
}

\begin{abstract}
Rivaroxaban is broadly used for the primary prevention of stroke and systemic embolism in the general population with non-valvular atrial fibrillation (AF). However, there is little published evidence on the safety and efficacy of rivaroxaban for AF in patients with active cancer. The aim of this study was to assess the safety and efficacy of rivaroxaban in patients with active cancer and AF. Use of rivaroxaban in cancer patients at Memorial Sloan Kettering Cancer Center (MSKCC) is monitored in the setting of a Quality Assessment Initiative. Patients with active cancer and AF, treated with rivaroxaban from 1/1/2014 through 3/31/2016 are included in this analysis. Clinical endpoints were defined a priori and assessed through text searches of medical records. A total of 163 evaluable patients were identified. After adjusting for competing risks, the estimated 1 year cumulative incidence of ischemic stroke was $1.4 \%(95 \% \mathrm{CI}=0-3.4 \%)$ and major bleeding was $1.2 \%(95 \% \mathrm{CI}=0-2.9 \%)$. The risk of clinically-relevant non-major bleeding leading to discontinuation of anticoagulation at 1 year was $14.0 \%$ (95\% $\mathrm{CI}=4.2-22.7 \%$ ). The cumulative incidence of mortality was $22.6 \%$ (95\% $\mathrm{CI}=12.2-31.7 \%$ ) at one year, reflecting an active cancer population. One patient died after developing an acute ischemic cerebrovascular insult. In
\end{abstract}

Corresponding author: Simon Mantha, MD, MPH, Memorial Sloan Kettering Cancer Center, 1275 York Ave, New York, NY 10065, Phone: (212)639-7573, Fax: (646)227-7246, manthas@ mskcc.org.

Publisher's Disclaimer: This is a PDF file of an unedited manuscript that has been accepted for publication. As a service to our customers we are providing this early version of the manuscript. The manuscript will undergo copyediting, typesetting, and review of the resulting proof before it is published in its final citable form. Please note that during the production process errors may be discovered which could affect the content, and all legal disclaimers that apply to the journal pertain. 
conclusion, the safety and efficacy of rivaroxaban treatment for non-valvular AF in patients with active cancer is comparable to the results of the ROCKET-AF study in the general population.

\section{Keywords}

anticoagulation; stroke; bleeding; cancer

The new direct oral anticoagulants (DOAC's) have been established as safe and effective alternatives to vitamin $\mathrm{K}$ antagonists (VKA's) in the general patient population, and within treatment recommendation guidelines for patients with non-valvular atrial fibrillation (AF). ${ }^{1-6}$ The ROCKET-AF study demonstrated the non-inferiority of rivaroxaban compared to VKA's for prevention of thromboembolism in patients with non-valvular AF. ${ }^{1}$ Patients with a serious concomitant illness associated with life expectancy of $<2$ years were excluded from this study, thereby limiting the extrapolation of those results to patients with active cancer. A post hoc pooled analysis of EINSTEIN PE and EINSTEIN DVT suggested that rivaroxaban appears to have reasonable efficacy and safety profiles for individuals with cancer-associated venous thromboembolism, as did single-institution cohort studies. ${ }^{7-9}$ The objective of this study was to assess the safety and efficacy of rivaroxaban in non-valvular $\mathrm{AF}$ patients with active cancer.

\section{Methods}

The use of rivaroxaban and associated clinical outcomes in patients with active cancer at Memorial Sloan Kettering Cancer Center (MSKCC) is monitored through an ongoing Quality Assessment Initiative (QAI). After approval by the Institutional Review Board (IRB), all rivaroxaban orders within the institution starting on January 1, 2014 were reviewed by the Hematology Service, and clinical data on these patients was entered into the QA database.

A Clinical Pathway Guideline (Appendix 1) for the use of rivaroxaban in cancer patients at MSKCC was derived at the end of 2013 by the Hematology/Anticoagulation Management Service. The Clinical Pathway addresses issues specific to the cancer population, including potential absorption defects, excretory organ function, drug interactions, thrombocytopenia, and bleeding risk. The recommended dose of rivaroxaban for AF is $20 \mathrm{mg}$ daily. ${ }^{1}$ For patients 75 years or older we recommended $15 \mathrm{mg}$ daily. We did not recommend rivaroxaban to patients with a creatinine clearance $<30 \mathrm{~mL} / \mathrm{min}$.

Patients with active cancer and AF treated with rivaroxaban from January 1, 2014 through February 14, 2016, were identified and are the subject of this analysis. Active cancer was defined as evidence of neoplasm on imaging, elevated levels of cancer markers on laboratory testing, or ongoing active cancer therapy (chemotherapy, hormone therapy, or radiation therapy). We also included patients if they were within 1 month of completion of adjuvant cancer therapy or within 1 month of cancer surgery. Patients with squamous cell cancer of the skin as their only malignancy, valvular disease (mitral stenosis, artificial heart valve, or mitral valve repair), or insufficient documentation were excluded from this analysis. ${ }^{10}$ Patients were included regardless of the primary indication for anticoagulation. 
To adjudicate the endpoints, clinical notes were analyzed using an automated text search. The terms used to perform the text search are shown in Appendix 2. All clinical notes containing at least one keyword were identified and further reviewed by a study investigator. Potential events identified by this first observer were further reviewed by a second physician investigator. All available non-MSKCC records were manually reviewed, since they were not subject to the text search.

Since the risk of bleeding on anticoagulation decreases with time and not accounting for this detail could result in bias, the analysis was performed accounting for the distinct acute and chronic phases of anticoagulation. The acute phase consisted of the first 90 days from initiation of any anticoagulant, while the chronic phase was defined as occurring after the first 90 days of anticoagulation. Some patients had initially been treated with another therapeutic anticoagulant (VKA, low-molecular-weight heparin or unfractionated heparin), before transitioning to rivaroxaban. Only events that occurred while the patients were on rivaroxaban are included in the analysis.

We conducted a time to event analysis. The following primary endpoints were defined $a$ priori: stroke, systemic embolism, major bleeding, death, and clinically-relevant non-major bleeding (CRNMB) leading to discontinuation of the drug for at least 7 days. Major bleeding was as defined by the International Society on Thrombosis and Haemostasis. ${ }^{11}$ For patients transitioning to hospice care, the date of transfer to hospice was considered the date of death due to the lack of clinical documentation after such a transfer.

We used a different definition of CRNMB than in ROCKET-AF. We only counted a CRNMB episode if it led to discontinuation of rivaroxaban for at least 7 days, while ROCKET-AF included as CRNMB instances of a patient contacting the study physician. March 31, 2016 was the last day of observation for this analysis. All patients who had not reached one of the above-mentioned endpoints were censored on that date. Patients were censored if they discontinued rivaroxaban for any reason other than reaching a predefined endpoint or transferred their care to another institution without further follow-up at MSKCC. Patients were also censored if they no longer had active cancer and were no longer receiving cancer directed therapy or if they were more than 3 months after their latest cancer surgery.

The cohort was considered left truncated and right censored, as several individuals had been exposed to anticoagulation before starting treatment with rivaroxaban. Survival analysis was performed by means of a counting process, whereas individual patients were observed only from the day they started rivaroxaban onward, having entered the cohort on the day they started anticoagulation with any agent. We performed competing risks analysis for stroke, major bleeding, death, and CRNMB leading to discontinuation of rivaroxaban for at least 7 days. The cumulative incidence was computed for each individual endpoint, treating the other three endpoints as competing risks. Additionally, standard univariate analysis was performed and pertinent covariates were entered in a Cox Proportional Hazards survival model. 


\section{Results}

A total of 163 patients with active cancer and AF treated with rivaroxaban were identified between January 1, 2014 and February 14, 2016. Baseline information is summarized in Table 1. The mean $\mathrm{CHA}_{2} \mathrm{DS}_{2}$-VASc was 3.2 and the mean $\mathrm{CHADS}_{2}$ score was 1.7. ${ }^{12} \mathrm{Six}$ patients had a $\mathrm{CHA}_{2} \mathrm{DS}_{2}$-VASc score of 0 . Three of these patients were on anticoagulation primarily for pulmonary embolism or a deep venous thrombosis, 2 were on anticoagulation for subsequent cardioversion, and 1 had already been on long-term anticoagulation with a VKA before transitioning to rivaroxaban.

The mean HAS-BLED score was $1.2 .{ }^{13}$ Because the INR is not pertinent to the use of rivaroxaban, the "labile INR" component from the original HAS-BLED score was not included. Therefore, the maximum points for this cohort for the HAS-BLED score was 8 instead of 9 . The majority of patients $(n=102)$ were already in the chronic phase ( $>90$ days) of anticoagulation. Fifty-nine patients were within the initial 90 days of anticoagulation (acute phase). In 2 cases the exact start date was not available, but was assumed to be within the last 90 days. In those 2 cases the observation start date was postponed by 90 days and they were included in the chronic cohort after 90 days.

Thirty-five patients reached a clinical endpoint during the observation period. Endpoints were stroke $(n=2)$, major bleeding $(n=2), C R N M B$ leading to discontinuation $(n=10)$, and death $(n=21)$. All the individuals with stroke or major bleeding had metastatic cancer, with lung/gastrointestinal and gynecological/breast origins for these two outcomes respectively. No systemic embolic events were identified. The mean observation period was 175 days (range 1 to 365 days). Fifty-four patients were followed up to their $365^{\text {th }}$ day of anticoagulation without reaching an endpoint.

Adjusting for competing risks and considering a one-year period, the estimated cumulative incidence for major bleeding was $1.2 \%$ (95\% $\mathrm{CI}=0-2.9 \%)$, CRNMB leading to discontinuation of rivaroxaban, $14 \%$ (95\% $\mathrm{CI}=4.2-22.7 \%)$, stroke, $1.4 \%$ (95\% $\mathrm{CI}=0-3.4 \%)$, and death, $22.6 \%$ (95\% CI=12.2-31.7\%) (Table 2 and Figure 1). There were no episodes of systemic embolism or hemorrhagic stroke. One patient died of a myocardial infarct concurrent with an acute ischemic cerebrovascular stroke.

A total of 74 patients were censored before reaching 365 days. Reasons for censoring included achievement of clinical remission $(\mathrm{n}=15)$, transfer of care to another institution $(n=8)$, loss to follow-up $(n=5)$, rivaroxaban discontinuation for unknown reasons $(n=2) .9$ patients elected to discontinue rivaroxaban, including 3 due to cost considerations. 24 patients stopped for medical reasons.

The medical reasons leading to discontinuation of rivaroxaban included: development of renal or liver dysfunction, thrombocytopenia, potential drug interaction between rivaroxaban and a newly added drug, change in digestive or urinary track after cancer surgery, admission for other medical reasons, and no further need of anticoagulation. One patient discontinued rivaroxaban and switched to a different anticoagulant after development of an upper extremity deep venous thrombosis. Eleven patients reached the end of observation date 
(03/31/16), prior to 365 days of anticoagulation. The mean observation time for these patients was 205 days (range 92 to 350 ).

A total of 123 invasive procedures were performed during the observation period. In 105 cases rivaroxaban was held for at least 24 hours. 3 patients underwent emergency interventions and rivaroxaban had not been held. Rivaroxaban was not held prior to intervention in one case, for unknown reason. No major bleed or ischemic stroke occurred during the surgeries or within 72 hours after the intervention. Eight patients were not restarted on rivaroxaban after their intervention due to medical reasons.

Nine episodes of moderate thrombocytopenia (platelet count $<50,000 / \mathrm{mcl}$ ) were recorded. The mean duration of thrombocytopenia was 11 days, ranging from 1 to 60 days. In 5 of these episodes the dose of rivaroxaban was not adjusted, although 4 of the 5 cases had only a single isolated platelet count $<50,000 / \mathrm{mcl}$ that resolved upon repeat testing. In 2 cases the drug was held transiently. One episode of thrombocytopenia was associated with a CRNMB leading to discontinuation of rivaroxaban.

Renal dysfunction (creatinine clearance $<30 \mathrm{ml} / \mathrm{min}$, Cockcroft-Gault) was observed in 5 patients (total of 8 episodes). ${ }^{14}$ Rivaroxaban was transiently held and then subsequently restarted in 3 episodes, discontinued in 1 case, and not adjusted in 4 cases. No major bleeding or stroke occurred during these episodes of renal dysfunction. Other events should be noted, although not included in the primary analysis. These include CRNMB episodes which did not lead to a discontinuation of rivaroxaban for 7 days or longer $(\mathrm{N}=24)$ and venous thromboembolic events $(\mathrm{N}=2)$.

Univariate analysis did not show any correlation between baseline values and CRNMB, but a correlation between cancer stage and death was observed as expected. A Cox Proportional Hazards model including age and cancer stage confirmed this association. Due to the low event rate of $\mathrm{MB}$ as well as stroke, no further analysis was conducted. Other events should be noted, although not included in the primary analysis. These include CRNMB episodes which did not lead to a discontinuation of rivaroxaban for 7 days or longer $(\mathrm{N}=24)$ and venous thromboembolic events $(\mathrm{N}=2)$.

\section{Discussion}

Cancer is associated with a hypercoagulable state as well as increased risk of bleeding. Those risks are not adequately captured by current stratification scores $\left(\mathrm{CHADS}_{2}\right.$ or $\mathrm{CHA}_{2} \mathrm{DS}_{2}$ VASc score). ${ }^{15}$ Nevertheless, our cancer cohort had similar $\mathrm{CHA}_{2} \mathrm{DS}_{2}$-VASc scores to the ROCKET-AF study; In the ROCKET-AF study $87 \%$ of the patients had a $\mathrm{CHA}_{2} \mathrm{DS}_{2}$-VASc score greater or equal to 3, compared to $69 \%$ for the MSKCC cohort.

There is no established guidance for anticoagulation management of AF, specific to patients with active cancer. DOAC's may be a reasonable anticoagulant choice in cancer patients, being much less sensitive to dietary changes or drug interactions than VKA's and less costly and burdensome than LMWH. Additionally, DOAC's have a shorter half-life than VKA's allowing for more rapid responses to transient episodes of thrombocytopenia or interruption for invasive procedures. ${ }^{16}$ 
Findings from this QAI program provide encouraging initial data on the safety and efficacy of rivaroxaban in cancer patients with AF. The safety and efficacy event rates in this study were similar to those reported in the general population in the ROCKET-AF trial. ${ }^{1}$ Mortality was substantial but largely attributable to underlying malignancy and lower than what was observed in the CLOT study which featured a similar population treated with dalteparin for venous thromboembolic disease. ${ }^{17}$ The cumulative incidence of stroke adjusting for competing risks was $1.4 \%$ at 1 year in this cancer cohort, similar to the $1.7 \%$ per year outcome for stroke and systemic embolism in the rivaroxaban group in the as-treated safety population of ROCKET-AF. ${ }^{1}$

The cumulative, 12 month rate of major bleeding in our cohort was $1.2 \%$. This compares favorably with the $3.6 \%$ rate in the rivaroxaban group in ROCKET-AF. ${ }^{1}$ The major bleeding rate was 5.1 per 100 patient years in the cancer arm of the ORBIT-AF registry. ${ }^{18}$ The cancer arm of the ORBIT-AF registry pooled patients with a VKA and dabigatran. Our cohort cannot be directly compared to ROCKET-AF, as ours is a retrospective cohort, and ROCKET-AF was a randomized, prospective clinical trial. Several sources of bias could account for a lower observed risk of major bleeding, including not accounting for transient periods off anticoagulation and the selection of a more favorable subset of patients by treating clinicians. But with these limitations in mind, we do not see evidence of trends towards inferior safety or efficacy with use of rivaroxaban for treatment of AF in patients with active cancer.

There are some other limitations to our cohort analysis. Rivaroxaban is cleared in the urine, and therefore our institutional guidelines recommend against use of rivaroxaban in patients with active gastrointestinal or genitourinary lesions, due to an expected high risk of bleeding in those sites. This could lead to selection bias and to a less vulnerable patient population up front.

This cohort also does not include all patients with active cancer and AF at MSKCC, as some individuals received anticoagulation care at the hands of their local internist or cardiologist, which in theory could affect the internal validity of our study. However, since the vast majority of MSK patients receive most or all of their care within the institution and our electronic records include documentation from outside medical encounters, this potential source of error would be minor.

\section{Supplementary Material}

Refer to Web version on PubMed Central for supplementary material.

\section{Acknowledgments}

Research support: Janssen Scientific Affairs, LLC

This research was funded in part through the NIH/NCI Cancer Center Support Grant P30 CA008748. 


\section{References}

1. Patel MR, Mahaffey KW, Garg J, Pan G, Singer DE, Hacke W, Breithardt G, Halperin JL, Hankey GJ, Piccini JP, Becker RC, Nessel CC, Paolini JF, Berkowitz SD, Fox KA, Califf RM. ROCKET AF Investigators. Rivaroxaban versus warfarin in nonvalvular atrial fibrillation. N Engl J Med. 2011; 365:883-891. [PubMed: 21830957]

2. Granger CB, Alexander JH, McMurray JJ, Lopes RD, Hylek EM, Hanna M, Al-Khalidi HR, Ansell J, Atar D, Avezum A, Bahit MC, Diaz R, Easton JD, Ezekowitz JA, Flaker G, Garcia D, Geraldes M, Gersh BJ, Golitsyn S, Goto S, Hermosillo AG, Hohnloser SH, Horowitz J, Mohan P, Jansky P, Lewis BS, Lopez-Sendon JL, Pais P, Parkhomenko A, Verheugt FW, Zhu J, Wallentin L. Aristotle Committees and Investigators. Apixaban versus warfarin in patients with atrial fibrillation. N Engl J Med. 2011; 365:981-992. [PubMed: 21870978]

3. Connolly SJ, Ezekowitz MD, Yusuf S, Eikelboom J, Oldgren J, Parekh A, Pogue J, Reilly PA, Themeles E, Varrone J, Wang S, Alings M, Xavier D, Zhu J, Diaz R, Lewis BS, Darius H, Diener HC, Joyner CD, Wallentin L. RE-LY Steering Committee Investigators. Dabigatran versus warfarin in patients with atrial fibrillation. N Engl J Med. 2009; 361:1139-1151. [PubMed: 19717844]

4. Giugliano RP, Ruff CT, Braunwald E, Murphy SA, Wiviott SD, Halperin JL, Waldo AL, Ezekowitz MD, Weitz JI, Spinar J, Ruzyllo W, Ruda M, Koretsune Y, Betcher J, Shi M, Grip LT, Patel SP, Patel I, Hanyok JJ, Mercuri M, Antman EM. ENGAGE AF-TIMI Investigators. Edoxaban versus warfarin in patients with atrial fibrillation. N Engl J Med. 2013; 369:2093-2104. [PubMed: 24251359]

5. January CT, Wann LS, Alpert JS, Calkins H, Cigarroa JE, Cleveland JC Jr, Conti JB, Ellinor PT, Ezekowitz MD, Field ME, Murray KT, Sacco RL, Stevenson WG, Tchou PJ, Tracy CM, Yancy CW. American College of Cardiology/American Heart Association Task Force on Practice Guidelines. 2014 AHA/ACC/HRS guideline for the management of patients with atrial fibrillation: a report of the American College of Cardiology/American Heart Association Task Force on Practice Guidelines and the Heart Rhythm Society. J Am Coll Cardiol. 2014; 64:e1-76. [PubMed: 24685669]

6. Kirchhof P, Benussi S, Kotecha D, Ahlsson A, Atar D, Casadei B, Castella M, Diener HC, Heidbuchel H, Hendriks J, Hindricks G, Manolis AS, Oldgren J, Popescu BA, Schotten U, Van Putte B, Vardas P. 2016 ESC Guidelines for the management of atrial fibrillation developed in collaboration with EACTS: The Task Force for the management of atrial fibrillation of the European Society of Cardiology (ESC)Developed with the special contribution of the European Heart Rhythm Association (EHRA) of the ESCEndorsed by the European Stroke Organisation (ESO). Eur Heart J. 2016; 37:2916-2919.

7. Prins MH, Lensing AW, Brighton TA, Lyons RM, Rehm J, Trajanovic M, Davidson BL, BeyerWestendorf J, Pap AF, Berkowitz SD, Cohen AT, Kovacs MJ, Wells PS, Prandoni P. Oral rivaroxaban versus enoxaparin with vitamin $\mathrm{K}$ antagonist for the treatment of symptomatic venous thromboembolism in patients with cancer (EINSTEIN-DVT and EINSTEIN-PE): a pooled subgroup analysis of two randomised controlled trials. Lancet Haematol. 2014; 1:e37-46. [PubMed: 27030066]

8. Bott-Kitslaar DM, Saadiq RA, McBane RD, Loprinzi CL, Ashrani AA, Ransone TR, Wolfgram AA, Berentsen MM, Wysokinski WE. Efficacy and Safety of Rivaroxaban in Patients with Venous Thromboembolism and Active Malignancy: A Single-Center Registry. Am J Med. 2016; 129:615619. [PubMed: 26797081]

9. Mantha S, Laube E, Miao Y, Sarasohn DM, Parameswaran R, Stefanik S, Brar G, Samedy P, Wills J, Harnicar S, Soff GA. Safe and effective use of rivaroxaban for treatment of cancer-associated venous thromboembolic disease: a prospective cohort study. J Thromb Thrombolysis. 2017; 43:166-171. [PubMed: 27696084]

10. Fauchier L, Philippart R, Clementy N, Bourguignon T, Angoulvant D, Ivanes F, Babuty D, Bernard A. How to define valvular atrial fibrillation? Arch Cardiovasc Dis. 2015; 108:530-539. [PubMed: 26184867]

11. Schulman S, Kearon C. Subcommittee on Control of Anticoagulation of the Scientific and Standardization Committee of the International Society on Thrombosis and Haemostasis. Definition of major bleeding in clinical investigations of antihemostatic medicinal products in nonsurgical patients. J Thromb Haemost. 2005; 3:692-694. [PubMed: 15842354] 
12. Lip GY, Nieuwlaat R, Pisters R, Lane DA, Crijns HJ. Refining clinical risk stratification for predicting stroke and thromboembolism in atrial fibrillation using a novel risk factor-based approach: the euro heart survey on atrial fibrillation. Chest. 2010; 137:263-272. [PubMed: 19762550]

13. Roldan V, Marin F, Manzano-Fernandez S, Gallego P, Vilchez JA, Valdes M, Vicente V, Lip GY. The HAS-BLED score has better prediction accuracy for major bleeding than CHADS2 or CHA2DS2-VASc scores in anticoagulated patients with atrial fibrillation. J Am Coll Cardiol. 2013; 62:2199-2204. [PubMed: 24055744]

14. Cockcroft DW, Gault MH. Prediction of creatinine clearance from serum creatinine. Nephron. 1976; 16:31-41. [PubMed: 1244564]

15. Farmakis D, Parissis J, Filippatos G. Insights into onco-cardiology: atrial fibrillation in cancer. J Am Coll Cardiol. 2014; 63:945-953. [PubMed: 24361314]

16. Soff GA. A new generation of oral direct anticoagulants. Arterioscler Thromb Vasc Biol. 2012; 32:569-574. [PubMed: 22345595]

17. Lee AY, Levine MN, Baker RI, Bowden C, Kakkar AK, Prins M, Rickles FR, Julian JA, Haley S, Kovacs MJ, Gent M. Randomized Comparison of Low-Molecular-Weight Heparin versus Oral Anticoagulant Therapy for the Prevention of Recurrent Venous Thromboembolism in Patients with Cancer (CLOT) Investigators. Low-molecular-weight heparin versus a coumarin for the prevention of recurrent venous thromboembolism in patients with cancer. N Engl J Med. 2003; 349:146-153. [PubMed: 12853587]

18. Melloni C, Shrader P, Carver J, Piccini J, Fonarow G, Ansell J, Gersh B, Go A, Hylek E, Herling I, Mahaffey K, Peterson E, Kowey P. Management and outcome of patients with atrial fibrillation and cancer: the ORBIT-AF registry. J Am Coll Cardiol. 2016; 67:727-727. 


\section{Cumulative Incidence for Competing Risks}

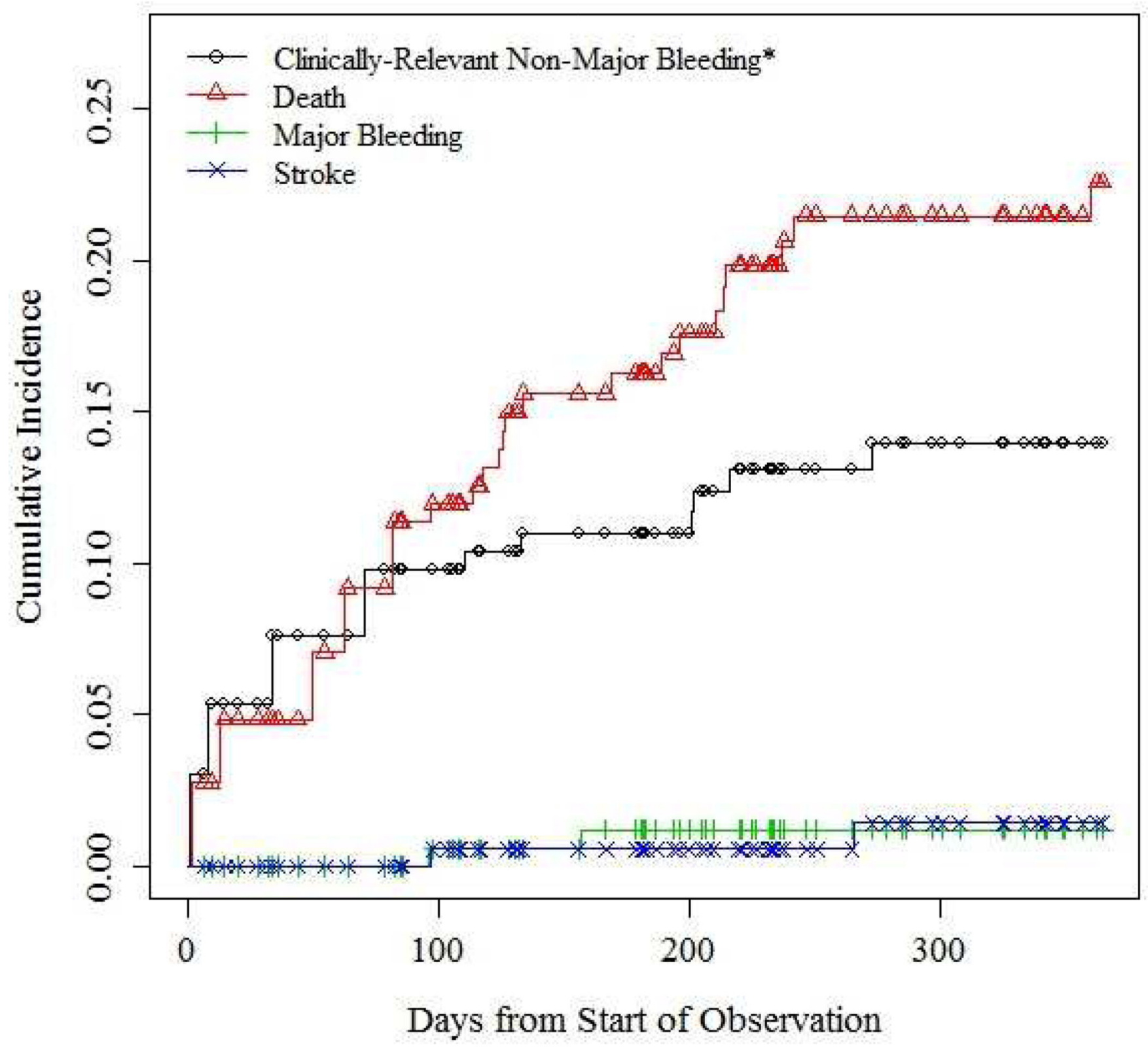

Figure 1.

Cumulative incidence for competing risks. *Clinically-relevant non-major bleeding leading to discontinuation of rivaroxaban for at least 7 days. 


\section{Table 1}

Baseline Characteristics of Patients

\begin{tabular}{|c|c|}
\hline Characteristic & \\
\hline Mean age in years (range) & $72(32-93)$ \\
\hline Women & $71(44 \%)$ \\
\hline \multicolumn{2}{|c|}{ Cancer stage of solid tumors* } \\
\hline I & $6(4 \%)$ \\
\hline II & $8(6 \%)$ \\
\hline III & $12(9 \%)$ \\
\hline IV & $81(59 \%)$ \\
\hline NED & $5(4 \%)$ \\
\hline Recurrent & $25(18 \%)$ \\
\hline \multicolumn{2}{|l|}{ Cancer type } \\
\hline Lung & $31(19 \%)$ \\
\hline Hematologic Malignancy & $24(15 \%)$ \\
\hline Gastrointestinal & $20(12 \%)$ \\
\hline Genitourinary & $18(11 \%)$ \\
\hline Breast & $16(10 \%)$ \\
\hline Other & $54(33 \%)$ \\
\hline \multicolumn{2}{|l|}{$\mathrm{CHA}_{2} \mathrm{DS}_{2}$-VASc Score } \\
\hline 0 & $6(4 \%)$ \\
\hline 1 & $12(7 \%)$ \\
\hline 2 & $32(20 \%)$ \\
\hline 3 & $55(34 \%)$ \\
\hline 4 & $31(19 \%)$ \\
\hline 5 & $15(9 \%)$ \\
\hline 6 & $7(4 \%)$ \\
\hline 7 & $4(2 \%)$ \\
\hline 8 & $1(1 \%)$ \\
\hline 9 & 0 \\
\hline \multicolumn{2}{|l|}{ Modified HAS-BLED Score ${ }^{\dagger}$} \\
\hline 0 & $18(11.1 \%)$ \\
\hline 1 & $96(58.9 \%)$ \\
\hline 2 & $40(24.5 \%)$ \\
\hline 3 & $9(5.5 \%)$ \\
\hline 4 to 8 & 0 \\
\hline
\end{tabular}

Creatinine Clearance $(\mathrm{mL} / \mathrm{min})^{*}$

$250 \quad 148(91 \%)$

$<50 \quad 10(6 \%)$

Unknown $\quad 5(3 \%)$

NED: no evidence of disease 
* Cancer staging was not applied to brain tumors and hematological malignancies.

tThe "labile INR" criterion was omitted.

${ }^{*}$ Creatinine clearance was estimated using the Cockroft-Gault formula. 14 


\section{Table 2}

Cumulative Incidence of Competing Risks for Patients in the Acute, Chronic and Combined Phases of Anticoagulation *

\begin{tabular}{llll}
\hline & $\begin{array}{l}\text { Acute Phase } \\
\mathbf{N = 5 9}\end{array}$ & $\begin{array}{l}\text { Chronic Phase } \\
\mathbf{N = 1 3 8}\end{array}$ & $\begin{array}{l}\text { Combined Period } \\
\mathbf{N = 1 6 3}\end{array}$ \\
\hline Ischemic Stroke (95\% CI) & 0 & $1.8 \%(0-4.3)$ & $1.4 \%(0-3.4)$ \\
Major bleeding (95\% CI) & 0 & $1.5 \%(0-3.6)$ & $1.2 \%(0-2.9)$ \\
Death (95\% CI) & $11.4 \%(1.4-20.3)$ & $14.2 \%(7.3-20.5)$ & $22.6 \%(12.2-31.7)$ \\
CRNMB (95\% CI) & $9.8 \%(0.2-18.4)$ & $5.4 \%(1.1-9.5)$ & $14.0 \%(4.2-22.7)$ \\
\hline
\end{tabular}

* Cumulative incidence estimates for the chronic phase are conditional to reaching day 90 of anticoagulation without sustaining an event. The chronic phase was defined as lasting 275 days and the combined period encompasses 365 days.

CRNMB: Clinically-relevant non-major bleeding leading to discontinuation of rivaroxaban for at least 7 days. 